\title{
Gas-generating TPGS-PLGA Microspheres loaded with Nanoparticles (NIMPS) for co-delivery of minicircle DNA and anti-tumoral drugs
}

Vítor M. Gaspar ${ }^{1}$, André F. Moreira. ${ }^{1}$, Elisabete C. Costa ${ }^{1}$, João A. Queiroz ${ }^{1}$, Fani Sousa ${ }^{1}$, Chantal Pichon ${ }^{2}$ and llídio J. Correia ${ }^{1 *}$

${ }^{1}$ CICS-UBI - Health Sciences Research Center, University of Beira Interior, 6200-506, Covilhã, Portugal

${ }^{2}$ Centre de BiophysiqueMoléculaire, CNRS UPR4301, Inserm and University of Orléans, 45071 Orléanscedex 02, France

${ }^{*}$ Corresponding author:

Prof. Dr. Ilídio J. Correia

Av. Infante D. Henrique,

6200-506 Covilhã, Portugal

Tel.: +351275329002

Fax +351275 329099

E-mail: icorreia@ubi.pt 


\section{Highlights:}

- Co-delivery of drugs-minicircle DNA by nanoparticle-in-microsphere multipart systems

- Gas-generating TPGS-PLGA microspheres present a pH-responsive drug release

- Inclusion of TPGS as coating for PLGA improved cellular uptake

- Minicircle DNA nanoparticles inside microspheres promote transgene expression

- Drug-gene loaded systems present higher cytotoxic activity in cancer cells

\section{Abstract}

Drug-DNA combination therapies are receiving an ever growing focus due to their potential for improvingcancer treatment. However, such approaches are still limited bythe lack ofmultipurposedelivery systems thatencapsulate drugs and condense DNA simultaneously. In this study, we describe the successfulformulation ofgas-generating $\mathrm{pH}$-responsiveD- $\alpha$ tocopherol PEG succinate-poly(D,L-lactic-co-glycolic acid)(TPGS-PLGA) hollow microspheresloaded with both Doxorubicin (Dox) andminicircle DNA (mcDNA)nanoparticles as a strategy to co-deliver these therapeutics. For this studymcDNA vectors were chosen due to theirincreased therapeutic efficiencyin comparison to standard plasmid DNA.The results demonstrate thatTPGS-PLGAmicrocarriers can encapsulate Dox and chitosannanoparticles completelycondense mcDNA. The loading of mcDNA-nanoparticles into microspheres was confirmed by 3D confocal microscopy and co-localization analysis. The resultingTPGSPLGA-Dox-mcDNA nanoparticle-in-microsphere hybridcarriersexhibit a well-defined spherical shapeand neutral surface charge.Microcarriersincubation in acidic $\mathrm{pH}$ produceda gasmediatedDox release,corroboratingthe microcarriers stimuli-responsive character.Also, the dual-loaded TPGS-PLGA particles achieved5.2-fold higher cellularinternalization in comparison with non-pegylatedmicrospheres. This increased intracellular concentration resulted in a highercytotoxic effect.Successful transgene expression was obtained after 
nanoparticle-mcDNA co-delivery in the microspheres.Overall these findingssupport the concept of using nanoparticle-microsphere multipart systemsto achieve efficient co-delivery of various drug-mcDNA combinations.

Keywords: Microspheres, Nanoparticles, Drugs, Minicircle DNA, Co-delivery 


\section{Introduction}

In the last decades the relativeineffectiveness of clinically administered anti-cancer therapeutics hashighlightedthenecessity todevelop novel treatments that can improve cancer prognosis and patient survival rates[1]. To deal with this unfavorable scenario efforts are being focused on the combinatorialadministration ofmultiple bioactive moleculessuch as: i.)anti-tumoral drugs, ii.) proteins, iii.) natural compounds, iv.) antibodies andv.) nucleic acids (e.g. DNA, RNA), as a strategy to achieve an improved therapeutic effectin comparison to stand-alone treatments[2]. Fromall combinatorialtherapies, thosebased on drug-DNA coadministration have shown to be particularly promising for targeting multiple cancer hallmarks[3-5].

Chemotherapy-nucleic acid combinations generally take advantage of drugs intrinsic antitumoral activity together withDNAcapacity to surpassadditionalgenetic abnormalities of cancer cells. Such coordinated action isexpectedtoresult in a synergistic effect, and ultimately, in tumor regression[5, 6].Despite its remarkable potential, thistreatment modality remainslimited bydrugssystemic cytotoxicity and by the establishment of cell drug resistance due toexocytosisvia drug efflux pumps[7].Adding to this fact,standard non-viral DNA expression vectors are unstable in blood circulationand relativelyineffective in promoting prolonged gene expressionin vivo[8, 9]. These factorsaffect the feasibility of co-administering free drugs and naked DNA through the various administration routes (e.g. intravenous, oral)and contribute for the reduced application of combinatorial therapies in the clinic.

A valuablestrategy to overcome thesedrawbacks is the formulation of micro and nanoparticlesthat can transpose biological barriers and deliver small molecule-gene combinations into unhealthy cells. To loadmultipletherapeutics simultaneously the delivery systems mustdisplay hydrophilic or hydrophobic character for drug encapsulation and positively charged groups for DNA condensation[10].Designing micro or nanocarriers with such versatileproperties remains highly challenging, and to date very few materialsexhibitedsuitable physicochemical properties to achieve this goal. In this context, 
Zhang and co-workers, used peptide-functionalized DSPE/PEG liposomes to perform the codelivery of Paclitaxel and microRNA antagomir-10b (miRNA-10b) in order to delay tumor growth and lung metastasis dissemination[11]. Recently,triblock copolymermicellar delivery systems for co-delivery of Doxorubicin (Dox) and minicircle DNA (mcDNA) have been developed in our group to attain an improved therapeutic effect [10]. mcDNA was selected, as exogenous gene expression vector, because it is devoid ofbacterial backbone, which is responsible for inducing transgene silencing and eliciting severe immunological responses from the host [12]. Besides, mcDNA provides higher levels of transgene expression both in vitro and in vivo in comparison with standard DNA[13]. While these drug-gene co-delivery studiesreveal a positive outcome from dual administration via nanodevices, the release of therapeutics was not actively controlled. Manipulating drug or gene release in a spatially and temporally controlled mode at the target siteis crucialfor further improvingcarriersbiological performance andmaximize the efficacy ofcombinatorial therapy[2, 14].

Stimuli-responsive carriersare aviable option to fulfill this purpose since cargo release can be promoted by different extracellular and intracellular signals[15, 16].Redox potential variation, enzymes, light, temperature and pHare some of the stimulus that can be exploited for creating dynamic delivery systems that sense and react to their surrounding environment $[17,18]$.Response to $\mathrm{pH}$ changes is a particularly valuable strategy for cancer therapy sinceacid-triggered release can be promoted at the tumor microenvironment and in cancer cells lysosomal compartments[19]. Gas-generating $\mathrm{pH}$-sensitive carriers are anadvanced class ofefficient stimuli-sensitive delivery systems that generate carbon dioxide gas $\left(\mathrm{gCO}_{2}\right)$, as a response to surrounding acidic environments. Such gas production is generally mediated by bicarbonate salts (sodium bicarbonate $\left(\mathrm{NaHCO}_{3}\right)$, or ammonium bicarbonate $\left.\left(\mathrm{NH}_{4} \mathrm{HCO}_{3}\right)\right)$, that are encapsulated inside the delivery systems. The $\mathrm{pH}$ responsiveness is conferred by the reaction between hydrogencarbonate anion $\left(\mathrm{HCO}_{3}^{-}\right)$with acid medium,whichin turnproduces $\mathrm{gCO}_{2}$ bubbles that trigger the formation of pores in particles shell and cargo release[19]. The therapeutic applicability of $\mathrm{gCO}_{2}$-based $\mathrm{pH}$ responsive systems was recently demonstrated by Liu and co-workers with the delivery of 
$\mathrm{NH}_{4} \mathrm{HCO}_{3}$ and Dox-loaded Cholesterol-mPEG $2000-\mathrm{DSPE}$ liposomes[20]. The delivery of $\mathrm{pH}$ sensitive liposomes to multi-drug resistant (MDR) breast cancer cells resulted in an increased intracellular drug concentration and improved cytotoxic activity[20]. To the best of our knowledge such gas-triggered delivery systemsare yet to be explored for dug-mcDNA combinatorial therapy.

Therefore, this study reports the successfulformulation of nanoparticle-in-microsphere hybrid delivery systems (NIMPS) based on pH-responsive TPGS-PLGA microspheres and amino acid modified chitosan nanoparticles as a strategy for promoting the dual delivery of mcDNA and Dox to cancer cells. In this multipart system amino acid-modified chitosan nanoparticles providecomplexation of mcDNA gene expression vectors.Modification of chitosan with amino acids, namely L-histidine and L-arginine,further grants the polymer backbone a pH-responsive capacity as our group previously reported[21]. This functionalization enhances nanocarriers biological performance, as well asgene expression efficiency[21], making it a good candidate for mcDNA delivery to cancer cells. The rationale of furtheremploying TPGS as a coatingfor gas-generating $\mathrm{PLGA}-\mathrm{NaHCO}_{3}$ microcarriers is established on previous evidence that this polymerenhances both cellular uptake and particles stability in physiological medium in comparison to standard poly(vinyl alcohol) (PVA) [22].Overall, our findings demonstrate that the use of FDA approved, TPGS and PLGA biodegradable polymers as materials for microparticles production assures the biocompatibility of the NIMPS system and its potential for translation to therapy.Moreover, NIMPSdemonstrated a gas-mediated $\mathrm{pH}$-sensitive drug release in acidic medium and an improved cellular uptake in cancer cells. Transgene expression mediated by mcDNA vectors was confirmed after nanoparticle delivery, corroborating the co-administration concept. As a final point,Dox delivery in NIMPS resulted in a cytotoxic effect comparable to that of free drug, a result that demonstrates the potential of using this multipart system for co-delivery of different mcDNA-chemotherapeutics combinations. 


\section{Materials and Methods'}

For a detailed description of the materials and methods the reader is referred to the electronic supplementary information.

\subsection{Methods}

\subsubsection{Preparation of Doxorubicin-loaded gas-generating hollow microspheres}

PLGA and TPGS-PLGA hollowmicrospheres were formulatedby using the water-in-oil-inwater double-emulsion solvent diffusion-evaporation method reported byKe and co-workers with slight modifications [19].Blank microparticles were prepared by the W/O/W method without Dox incorporation in the first aqueous phase. Dox-loaded microspheres with PVA or TPGS coating are termed micro-PVA-Dox and micro-TPGS-Dox, respectively.

\subsubsection{Preparation of mcDNA Amino acid-Chitosan nanoparticles}

Prior to preparation of chitosan-mcDNA nanoparticles the cationic biopolymer was chemically modified with L-arginine and L-histidinevia EDC/NHS coupling chemistry to yield multifunctional Chitosan-Arginine-Histidine ( $\mathrm{CH}-\mathrm{HR}$ )as our group previously reported[21].All nanoparticles were formulated at the amine-to-phosphate ratio of 60 (N/P 60) since this formulation has previously presented optimal stability, biological performance and transfection efficiency [21]. mcDNA complexation by $\mathrm{CH}-\mathrm{HR}$ was confirmed by agarose gel electrophoresis [23]. Nanocarriers loading into Micro-TPGS-Dox microspheres was accomplished by adding $\mathrm{CH}-\mathrm{HR}$-mcDNA nanoparticles in the primary aqueous phase of the W/O/W assembly process. The resulting Micro-TPGS-Dox-(CH-HR-mcDNA) nanoparticle-inmicrosphere systems are designated by NIMPS from herein onwards.

\subsubsection{Delivery systems physicochemical characterization}

Microspheres and nanoparticlessizewasanalysed by Dynamic Light Scattering (DLS) in a ZetasizerNanoZS (Malvern Instruments, Worcestershire, UK). Three independent measurements were performed indisposable folded capillary cells (DTS1070), at 25드, and with a $173^{\circ}$ scattering angle.Microspheres morphology wasevaluated by scanning electron 
microscopy (SEM) by using a Hitachi S-3400N scanning electron microscope(Hitachi, Japan).The existence of the gas-generating agent $\left(\mathrm{NaHCO}_{3}\right)$ in PLGA microspheres core was determined by $X$-ray diffraction spectroscopy (XRD).

\subsubsection{Drug encapsulation and in vitro release}

Drug encapsulation efficiency in allPLGA microsphere formulations was determined through UV-vis spectrophotometry at $\lambda=485 \mathrm{~nm}$ by using aShimadzu-1700spectrophotometer (Shimadzu Inc., Japan). Drug encapsulation efficiency was calculatedas reported in the literature[24].Drug release from microspheres was evaluated by the dialysis method, in duplicate independent experiments. Drug release was carried out at $37^{\circ} \mathrm{C}$ in a water bath, with magnetic stirring $(500 \mathrm{rpm})$, in the dark. Supernatant samples were collected at various time-pointsand the drug content was analyzed by UV-vis spectrophotometry.

\subsubsection{Cellular uptake}

The cellular uptake of drug-loaded microparticles (micro-PVA-Dox, micro-TPGS-Dox) and Dox-mcDNA dual loaded NIMPSwas evaluated by flow cytometry. This analysis was carried out in a BD FACSCalibur flow cytometer (Becton Dickinson Inc., USA). Data acquisition was performed with the CellQuest software where $1 \times 10^{4}$ events were collected in the region of interest assigned toHeLa cells. Additionally, particles cellular uptake was visualized by confocal laser scanning microscopy (CLSM). Confocal images were acquired in a Zeiss LSM 710 confocal microscope (Carl Zeiss SMT Inc., USA). 3D reconstruction, colocalization analysis and image processing was performed in Zeiss Zen 2010, ImageJ and Imaris software (Bitplane, Switzerland).

\subsubsection{In vitro gene expression in $2 \mathrm{D}$ cultures}

mcDNA-GFP expression in cancer cells was evaluated by spectrofluorimetry[23].For this assay,HeLa cells were cultured in 96 well black-clear bottom plates at a density of $1 \mathrm{x}$ $10^{4}$ cells per well. Gene expression was quantified after 48 hby using a plate 
readerspectrofluorometer(Spectramax Gemini XS, Molecular Devices LLC, USA), at $\lambda_{\mathrm{ex}}=488$ $\mathrm{nm}$ and $\lambda_{\mathrm{em}}=519 \mathrm{~nm}$.In addition, GFP expression was also visualized and quantifiedby CLSM by using a Zeiss LSM 710 confocal microscope (Carl Zeiss SMT Inc., USA), equipped with a Plan-Apochromat $63 x / 1.4$ oil objective.For all experiments, 3D z-stacks of randomly distributed cell fields ( $n=3$ ) were acquired. GFP fluorescence intensity in cells volume was quantified by using the Imaris software(Bitplane, Switzerland).

\subsubsection{Cytotoxic activity}

The cytotoxic activity of Dox-loaded microspheres and mcDNA-Dox dual loaded NIMPSwas evaluated by the MTS assay as previously described [10]. Dead cells (70\% EtOH), and nontreated cells were used as positive and negative controls, respectively.

\subsubsection{Statistical analysis}

One-way analysis of variance (ANOVA) with the post-hoc Newman-Keuls test were used for statistical analysis of three or more groups. Student's t-test was used for comparison between two groups. A p value below 0.05 was considered statistically significant. 


\section{Results and Discussion}

\subsection{Formulation of Gas-generating PLGA Microspheres}

Gas-generating $\mathrm{pH}$ responsive hollow PLGA microspheres were produced by the double emulsion water-on-oil-water (W/O/W) solvent diffusion-evaporation methodas schematized in supplementary Figure S1A. Initially thefirstwater phase comprised by the gas-generating agent (sodium bicarbonate, $\left(\mathrm{NaHCO}_{3}\right)$ )and bythe PVA surfactant, were mixed with the oil phase containing PLGA dissolved in dichloromethane. Primaryemulsification was promoted by the action of ultrasound waves which generated well-defined PLGA droplets by disrupting the oil-water interface.

The resulting microcarriers were subsequently mixed with the second water phase comprised either by PVA or TPGS (Figure S1A). TPGS was used as an alternative to PVA for microspheres production because the PLGA-NaHCO $3-\mathrm{PVA}-\mathrm{PVA}$ systemsgenerally described in the literature have shown relatively low cellular uptake and also reduced stability in biological fluids [25]. Thus,as a strategy to improvemicrocarriers biological performance, TPGS was used as the surfactant in a second emulsification that involves high speed homogenization (supplementary Figure $\mathrm{S} 1 \mathrm{~A}$ ). Following the establishment of the W/O/W double emulsion the organic solvent was diffused by mixing and evaporated under reduced pressure which led to the formation of spherical PLGAmicrospheres coated with TPGS or PVA(supplementary Figure S1B and D). The produced micro-TPGS carriers had lowerpolydispersity than micro-PVA formulations,suggestingthat the coating process yields more homogeneous particles when this surfactant is used in the end of the process. MicroTPGS microspheres also exhibit a slightlylarger size when compared to their PVA counterparts a finding that can be attributed to the PEG shell that is formed in particles surface. Particle surface charge characterizationshowsthat the inclusion of TPGS causes a slight decrease in zeta potential(supplementary Figure S1C and E). These findings are in accordance with those obtained by $\mathrm{Mu}$ and co-workers which studied the impact of different surfactants in Paclitaxel-loaded PLGA nanoparticles. The inclusion of TPGS resulted in 
larger sized nanocarriers with more negative surface charge in comparison with those formulated PVA [26].

Following the successful formulation of TPGS coated PLGA microspheres the presence of the gas-generating agent in the carriers was analyzed by X-ray diffraction. As demonstrated in Figure $2 \mathrm{~A}$ thecharacteristic peaks of $\mathrm{NaHCO}_{3}$ crystalline structureare presentin microsphere formulations, indicating the effective encapsulation of the bicarbonate salt in particles during the assembly process. The broad XRD peak centered at $2 \Theta \sim 19.5^{\circ}$ is assigned to amorphous PLGA microspheres (Figure 1A). Interestingly, no significant differences in $\mathrm{NaHCO}_{3}$ characteristic diffraction peaks is observed between micro-PVA and micro-TPGS formulationssuggesting that the addition of TPGS in the end of the process does not affect $\mathrm{NaHCO}_{3} \quad$ encapsulation.
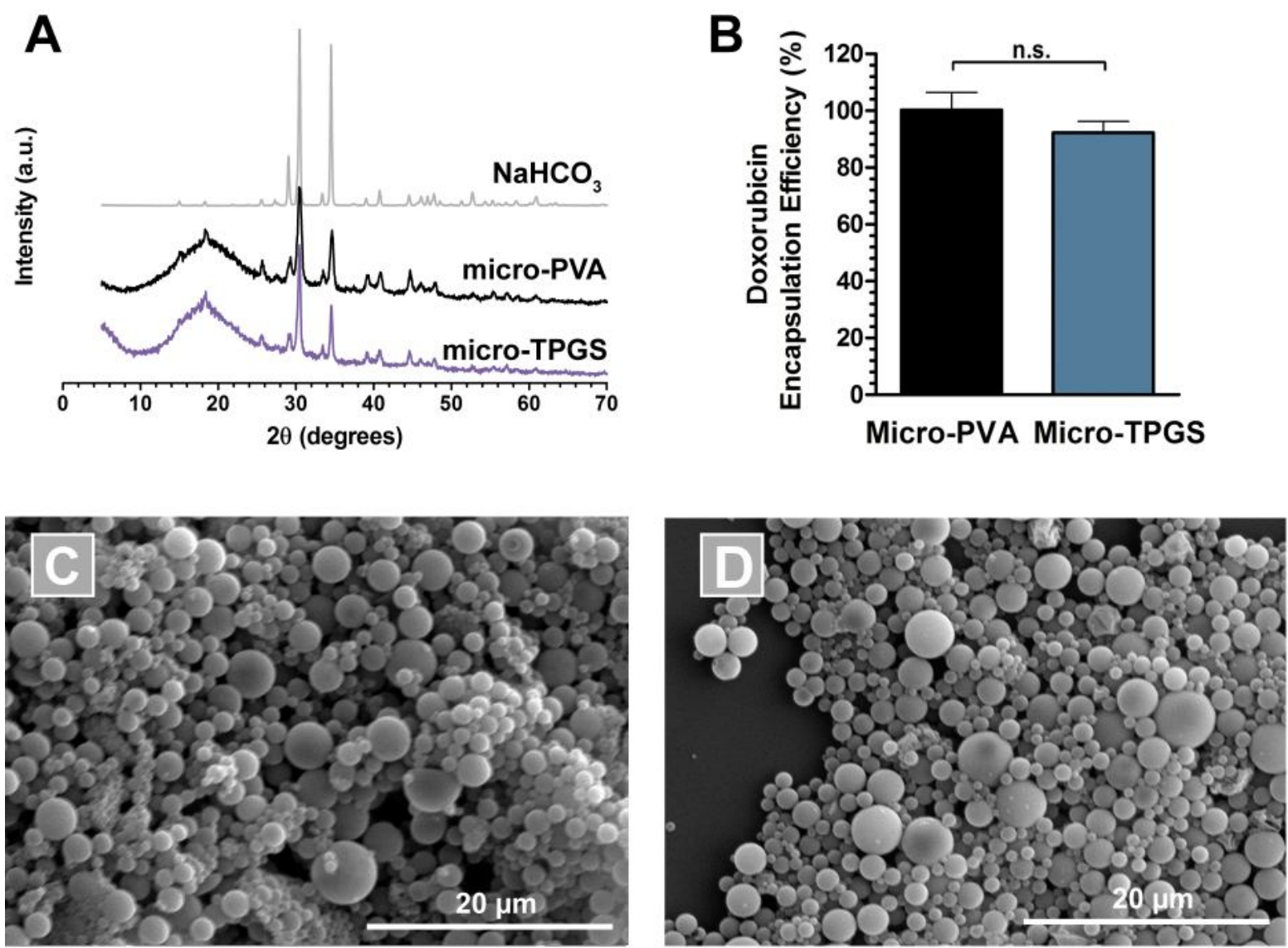

Figure 1.Microcarriers physicochemical characterization.A.) XRD analysis of microsphere formulations. B.) Dox encapsulation in different microsphere formulations. Data is presented 
as mean \pm s.d., $n=3$. $C$ and D.) SEM micrographs of micro-PVA-Dox and micro-TPGS-Dox formulations, respectively.

The potential of PLGA gas-generating microspheres to encapsulate anti-tumoral drugs was evaluated by including Doxorubicin (Dox) in the first aqueous phase of the assembly process. As the results in Figure $1 \mathrm{~B}$ demonstrate the encapsulation efficiency for both formulations is higher than $95 \%$ and no significant difference between micro-TGPS-Dox and micro-PVA-Dox particles is obtained. Drug encapsulation in particles core also had negligible influence in particles morphology. In fact,as observed in SEM micrographs all formulations maintained their characteristic spherical shape (Figure $2 \mathrm{C}$ and $\mathrm{E}$ ). Interestingly, the physicochemical characterization of drug loaded microspheres reveals that encapsulation of Dox in micro-TPGS carriers leads to the production of smaller particles (z-average size $=$ $1877 \mathrm{~nm}$ ) when compared to blank TPGS-based formulations (z-average size $=2362 \mathrm{~nm}$ ) (supplementary Figure S2A and B).Microspheres surface charge is also changed upon drug encapsulation in micro-PVA-Doxand micro-TPGS-Dox formulations, with the latter presenting a neutral zeta potential $(\zeta=-9.17 \mathrm{mV})$. This neutrality is highly important for the therapeutic efficiency of micro or nanoparticle formulations since it has been previously demonstrated that particles zeta potential in the range of neutrality $(+10 \mathrm{mV}$ to $-10 \mathrm{mV})$ achieve optimal for tumor penetration [27].

\subsection{Microcarriers biological characterization}

To further characterize the biological properties of micro-sized delivery systems, in vitro biocompatibility assays were performed in HeLa cells. The obtained results show that HeLa cells remain metabolically active up until $72 \mathrm{~h}$ when incubated with micro-PVA or microTPGSparticles (Supplementary Figure S3).

The cellular uptake capacity of bothformulations in physiological conditions was also evaluated by using flow cytometry. The obtained resultsdepicted in Figure $2 \mathrm{~A}$ indicate that micro-PVA-Dox carriers exhibit a cellular uptake comparable to that of free drug.In addition, micro-TPGS-Dox achieve approximately a 5.2-fold higher cellular uptake in comparison to 
their micro-PVA-Dox counterparts (supplementary Figure S4 A and B), indicating that the inclusion of TPGS enhances the biological efficiency of gas-generating PLGA microspheres. These results are in agreement with those recently reported by Kulkarnu and Feng, 2013, in which the inclusion of TPGS in polystyrene particles surface has shown to significantly increase cellular uptake in comparison with unmodified particles [28].
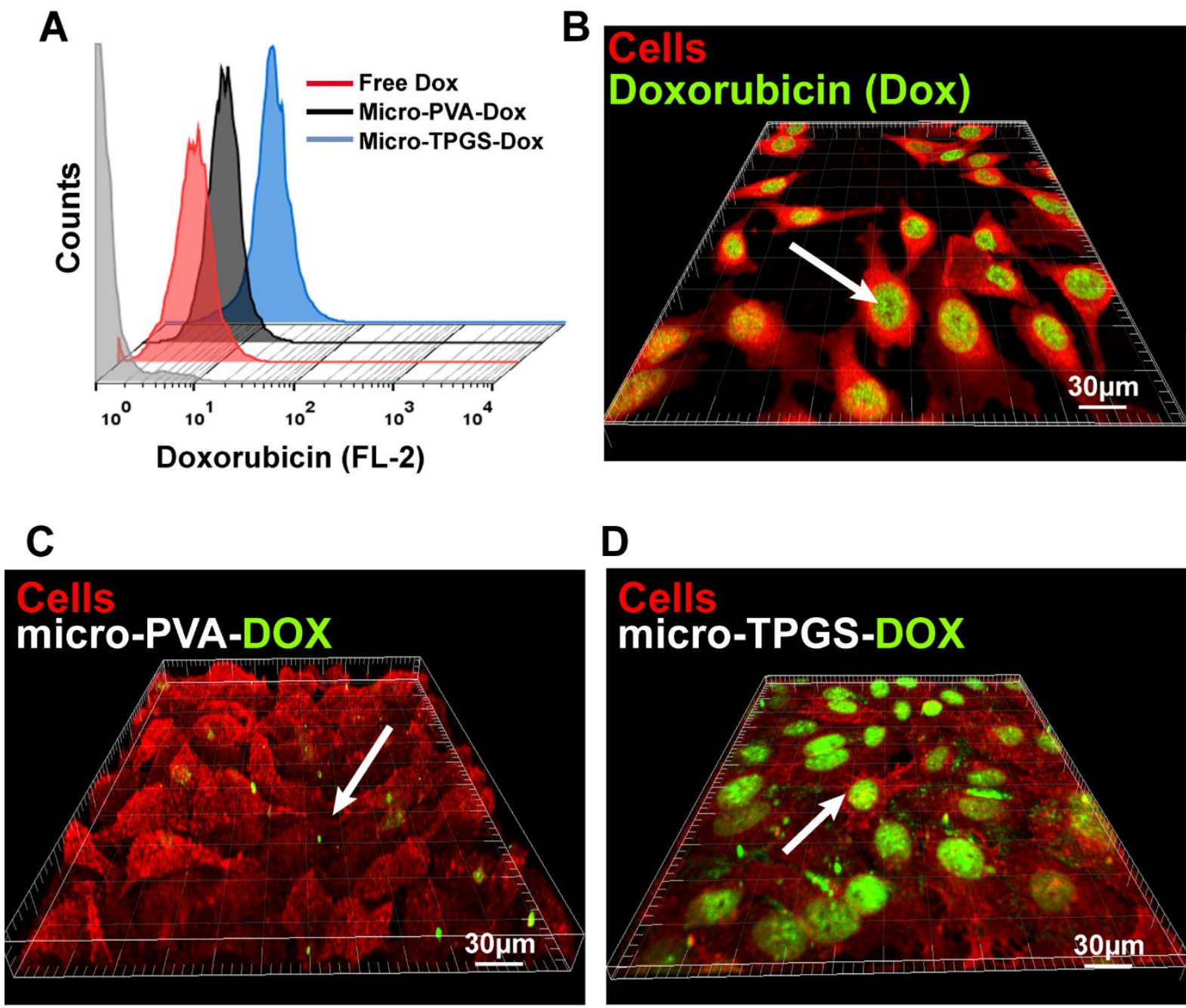

Figure 2. Analysis of drug loaded microspheres uptake in HeLa cancer cells. A.) Representative histograms of microcarriers and free drug cellular uptake. CLSM visualization of free Dox (B.), micro-PVA-Dox microspheres (C.), micro-TPGS-Dox microspheres (D) cellular uptake and intracellular localization. Red channel: WGA-Alexa Fluor ${ }^{\circledR} 594$ conjugate; Green channel: Doxorubicin. 
Thecellular uptake and intracellular localization of Dox loaded microcarriers was additionallyconfirmed by confocal microscopy. As depicted in Figure 2 B the administration of free drug leads to its intracellular accumulation particularly in the nuclear compartment (white arrows). In contrast, micro-PVA-Dox exhibit a widespread distribution in the intracellular compartment and few cells display Doxin the nuclear compartment (Figure $2 \mathrm{C}$, white arrows and supplementary figure S4, D1 and D2). The micro-TPGS-Dox carriers also have a random intracellular distribution(white arrows), however, a clear accumulation of the antitumoral drug in the nuclear compartment is visible (Figure 2D, white arrows; supplementary figure S4, E1 and E2). We hypothesize that such difference is correlated with the enhanced cellular uptake efficiency of micro-TPGS-Dox carriers. From a therapeutic point of view achieving this increased intracellular concentration and nuclear localization is critical for improving the overall therapeutic efficacy as Dox intracellular targets are mainly localized in the nuclear compartment[29]. The cellular uptake of microcarriers with this hydrodynamic size has also been reported in the literature[25]. In fact, the interesting work of Ke et al, explored the cellular uptake routes of PLGA-PVA microspheres, revealing that this event is an energy dependent process and that macropynocytosis plays a significant role in microcarriers internalization [25].

\subsection{Stimuli-responsivedrug release}

Since TPGS-based microcarriers present an improved cellular uptake efficiency, their responsiveness to acidicmilieu was investigated. 


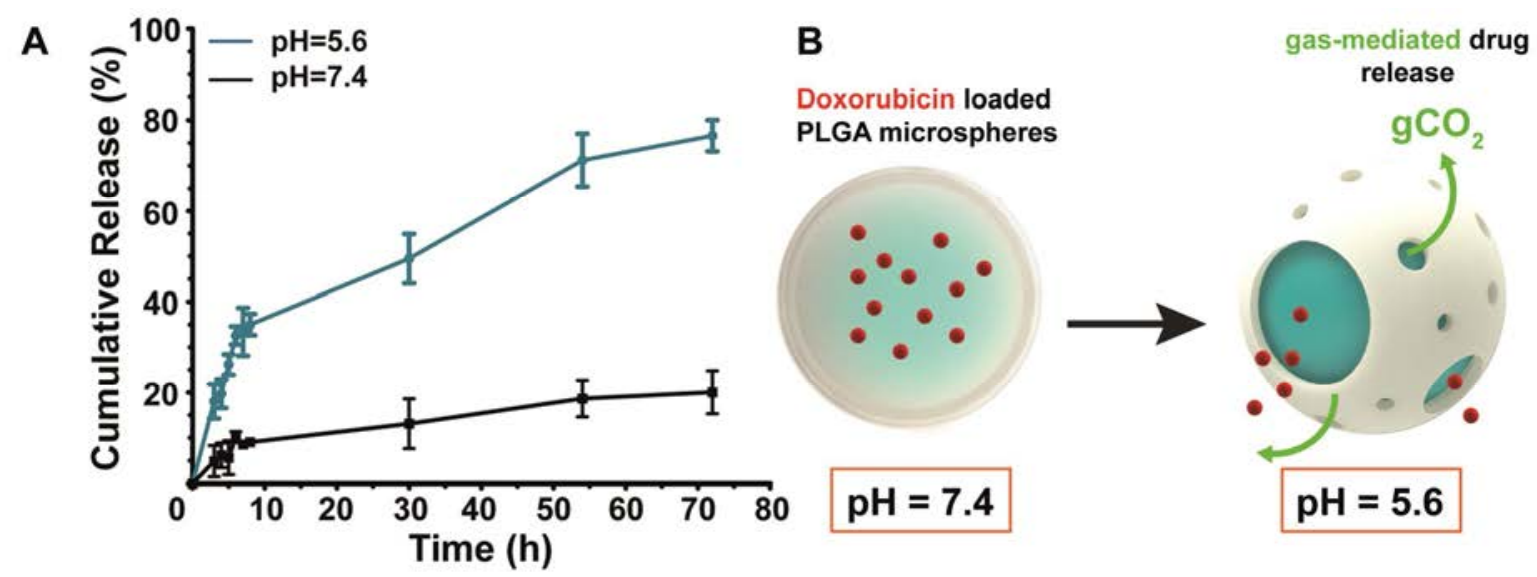

Figure 3. Analysis of drug release from pH-responsive TPGS-coated PLGA microspheres. A.) Cumulative Dox release at physiological and acidic pH.B.) Schematics that depicts the underlying principle of gas-generation in acidic environment with subsequent particles shell disruption and drug release.

The stimuli-dependent release is promoted via a $\mathrm{pH}$-triggered disruption of particles coreshell structure (schematics of Figure 3 B).Such responsiveness of micro-TPGS-Dox microcarriers is governed by the generation of $\mathrm{gCO}_{2}$ bubbles that are produced via reaction of sodium bicarbonate with acid $\left(\mathrm{NaHCO}_{3}+\mathrm{HCl} \rightarrow \mathrm{NaCl}+\mathrm{H}_{2} \mathrm{CO}_{3} ; \mathrm{H}_{2} \mathrm{CO}_{3} \rightarrow \mathrm{CO}_{2(\mathrm{~g})}+\mathrm{H}_{2} \mathrm{O}\right)[30]$. Eventually the resulting $\mathrm{gCO}_{2}$ bubbles diffuse through particles shelland originatepores whichstimulate a rapid release of the anti-tumoral drug (Figure 3 B).Drug release assays were performed both in physiological and acidic conditions in an attempt to mimic a stimulicontrolled drug release in the acidic tumor microenvironment or acidic cell compartments. As the results demonstrate the immersion of TPGS-coated PLGA microspheres in acidic medium results in a rapid and extensive Dox release (Figure $3 \mathrm{~A}$ ). In fact, the particles that were exposed to $\mathrm{pH}=5.6$ exhibit a 3.88 -fold higher drug release at $8 \mathrm{~h}$, when compared to their equivalents immersed in physiological conditions $(\mathrm{pH}=7.4)$ (Figure 4A). This tendency is also maintained after $70 \mathrm{~h}$ of incubation. It is important to emphasize that at physiological $\mathrm{pH}$ the microcarriers have negligible burst releasesince only $13 \%$ of loaded drug is released at $30 \mathrm{~h}$ (Figure $3 \mathrm{~A}$ ). Retention of the anti-tumoral drug in the particles core is crucial to reduce off-target cytotoxic effects upon their administration in vivo[14]. 
Additionally, SEM imaging was used to complement the characterization of microsphere structural stability in environments with different $\mathrm{pH}$. The results in supplementary Figure S5show that micro-TPGS carriers preserve their structural and morphological integrityupon incubation in physiological conditions, whilemicrospheres exposed to acidic mediumdisplay various holes in their shell due to the effervescent reaction promoted by $\mathrm{NaHCO}_{3}$ and acid (White square, supplementary Figure S5B2 and B3). Particles disintegration is also confirmed by the significant amount of free polymer present in SEMmicrographs (supplementary Figure S5 B1 to B3, white arrows).Such findingsconfirm the stimuliresponsive profile ofmicro-TPGS particles,contributing for the envisioned control overdrug release at the target tumor site.

\subsection{Formulation of drug-gene loaded pH-responsive PLGA Microspheres (NIMPS)}

Since TPGS-coated microcarriers demonstrate a pH-responsive profile and also an improved cellular uptake, the co-delivery of drugs and genes was investigated by combining microTPGS-Dox with mcDNA-loaded amino acid chitosan ( $\mathrm{CH}-\mathrm{HR}$ ) nanoparticles as a strategy to promote the dual delivery of these therapeutics (schematics of Figure 4). 
A

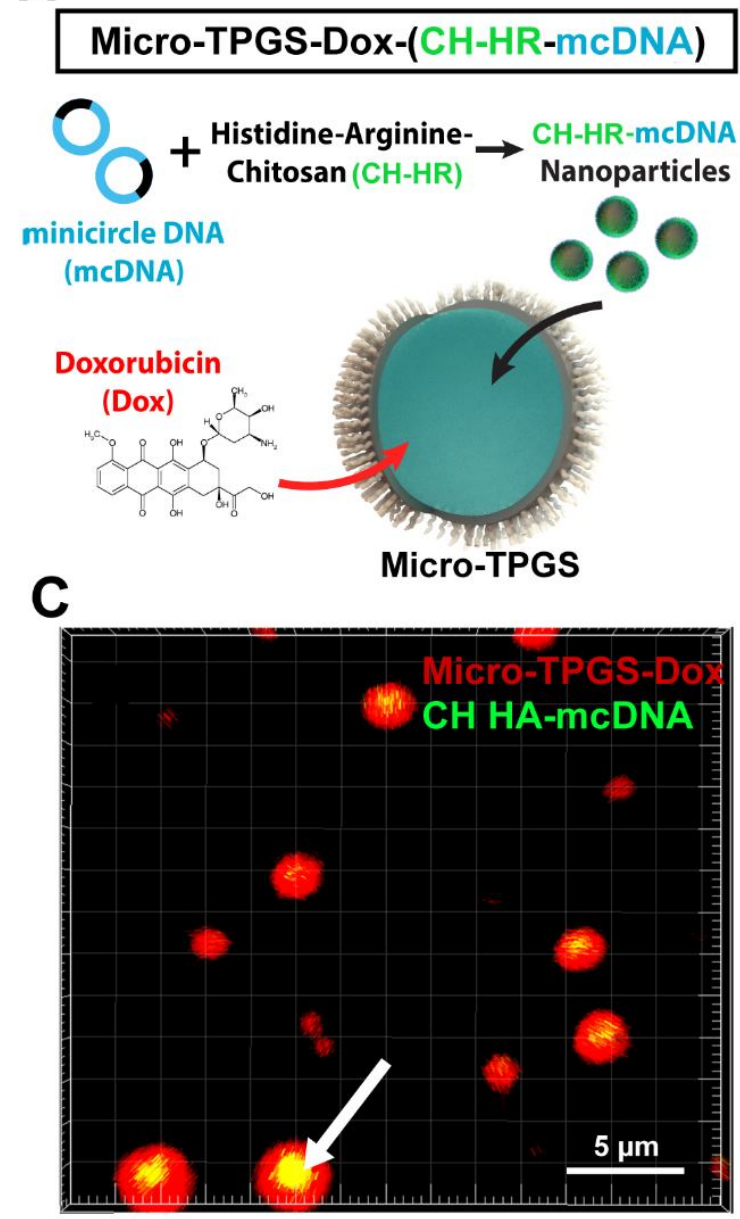

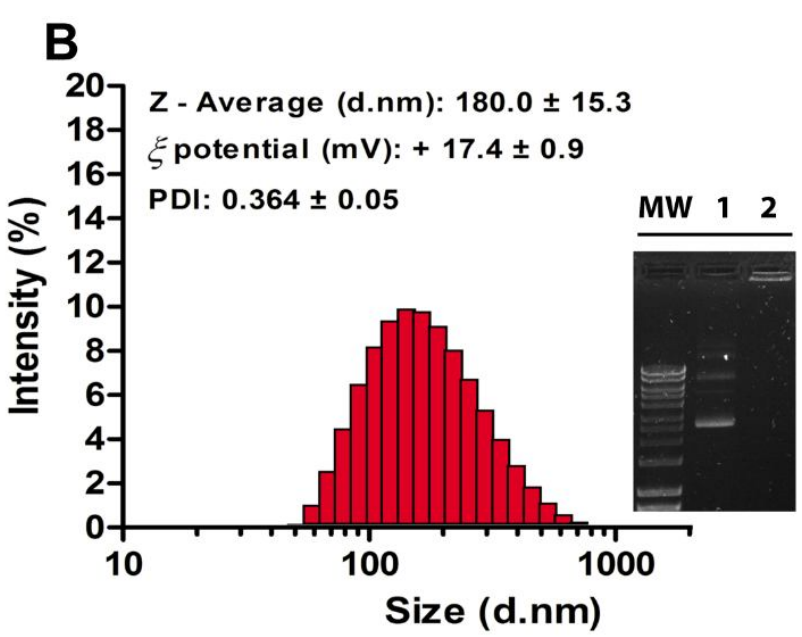

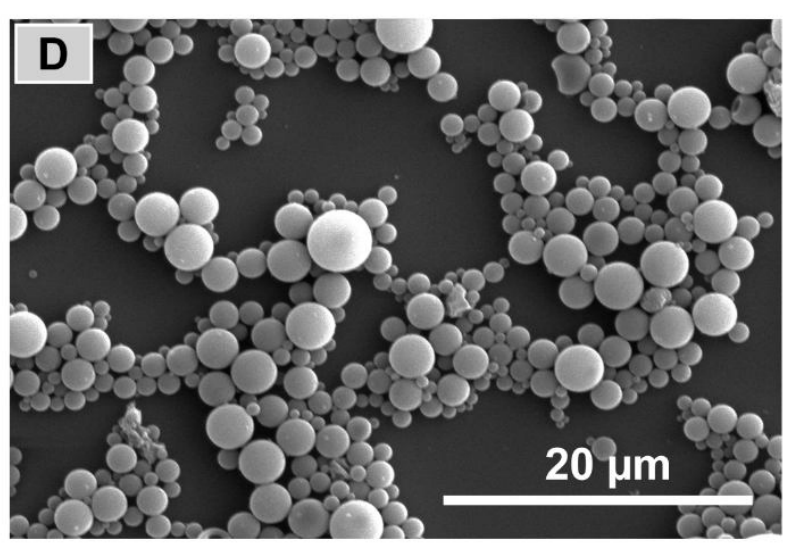

Figure 4. Production of nanoparticle-in-microsphere (NIMPS) hybrid delivery systems. A.)

Schematics of the hybrid delivery systems comprised by micro-TPGS-Dox and CH-HRmcDNA loaded nanoparticles (not drawn in scale). B.) DLS and agaroseelectrophoretic analysis of DNA minicirclescondensation and CH-HR-mcDNA nanoparticles assembly.C.) Confocal microscopy micrographs of NIMPS dispersed in an imaging chamber.Red channel: Doxorubicin encapsulated in microspheres. Green channel: FITC-labelled mcDNA. Yellow channel: merged red and green channels. White arrows indicate FITC-mcDNA.D.) SEM micrograph of NIMPS.

Prior to NIMPS formulation the production of $\mathrm{CH}-\mathrm{HR}-\mathrm{mcDNA}$ nanoparticles was investigated. mcDNA chitosan nanoparticles were assembled via electrostatic attraction between the negatively charged phosphate groups of mcDNA and the cationic groups in the polymer backbone under mild conditions. The amino acid modification of chitosan with Larginine and L-histidine ( $\mathrm{CH}-\mathrm{HR})$, also contributes for the condensation of genetic material, 
namely plasmid DNA (pDNA), as our group previously demonstrated [21]. In this study, this improved chitosan derivative has also shown to completely condense novel mcDNA vectors as evidenced by agarose gel electrophoresis (Figure $4 \mathrm{~B}$ ). These findings assume further importance since few reports have explored mcDNA vectors condensation by cationic biomaterials so far $[10,31,32]$. The resulting mcDNA nanoparticles have an average size below $200 \mathrm{~nm}$ and a positive zeta potential (Figure $4 \mathrm{~B}$ ).

The production of NIMPS was carried out by including CH-HR-mcDNA nanoparticles in the first aqueous phase. The resulting hybrid delivery systemsexhibit an average size of 2502 $\mathrm{nm}$ and are homogeneous (PDI: 0.208, supplementary Figure S6 A). The dual loaded carriers also display a less negative zeta potential ( $\zeta:-1.89 \pm 0.2 \mathrm{mV})$ when compared to single loaded micro-TPGS-Dox formulations ( $\zeta:-9.17 \pm 0.6 \mathrm{mV}$ ) (supplementary Figure S6 A). This increase in zeta potential corroborates the successfulencapsulation of positively charged nanoparticles in microcarriers core. The resulting NIMPS alsomaintain their spherical morphology (Figure $4 \mathrm{D}$ ), indicating that theaddition of nanoparticles does not significantly affect microspheres assembly.

Toconfirm the presence of mcDNA nanoparticles inside the PLGA microcarriers, the dual system was also analyzed by confocal microscopy. As displayed in Figure $4 \mathrm{C}$ the fluorescence signal corresponding to mcDNA-FITC nanoparticles is coincident with microTPGS-Doxcarriers. This fact is supported by co-localization analysis (Supplementary Figure S7). A 3D reconstruction of microspheres volume further confirmed the presence of mcDNAloaded nanoparticles in microspheres interior (supplementary Figure S6 B).

\subsection{NIMPS cellular uptake and gene expression}

The cellular uptake analysis of NIMPS demonstrates that the hybrid carriers exhibit higher internalization in cancer cells $(87.1 \pm 4.1 \%$ cells $)$ in comparison to micro-TPGS-Dox counterparts (72.9 $\pm 6.1 \%$ cells) (supplementary Figure S8 A). Confocal microscopy images 
additionally confirmed that NIMPS are localized in the intracellular compartment and that Dox is present in cancer cells nucleus (supplementary Figure S8 B, white arrows).

To complement NIMPS characterization the gene expression mediated by mcDNA-GFP when co-delivered with Dox was assessed. The results demonstrate that GFP expression achieved by $\mathrm{CH}-\mathrm{HR}$-mcDNA nanoparticles is slightly higher thanthatobtained with NIMPS. However, this difference is not significant (supplementary Figure S8 E and F), and may be attributed to the fact that mcDNA-nanoparticles must be released from microcarriersbefore being localized in the cytoplasmas emphasized by Shardool and co-workers that developed nanoparticle-in-microsphere systems for oral delivery[33]. Besides, these results demonstrate that mcDNA-nanoparticles delivered in NIPMS achieve successful GFP expression.

\subsection{Delivery systems cytotoxic activity}

The cytotoxic activity of both single and dual-loaded delivery systems produced throughout this study was investigated to provide evidence aboutNIMPS therapeutic potential.

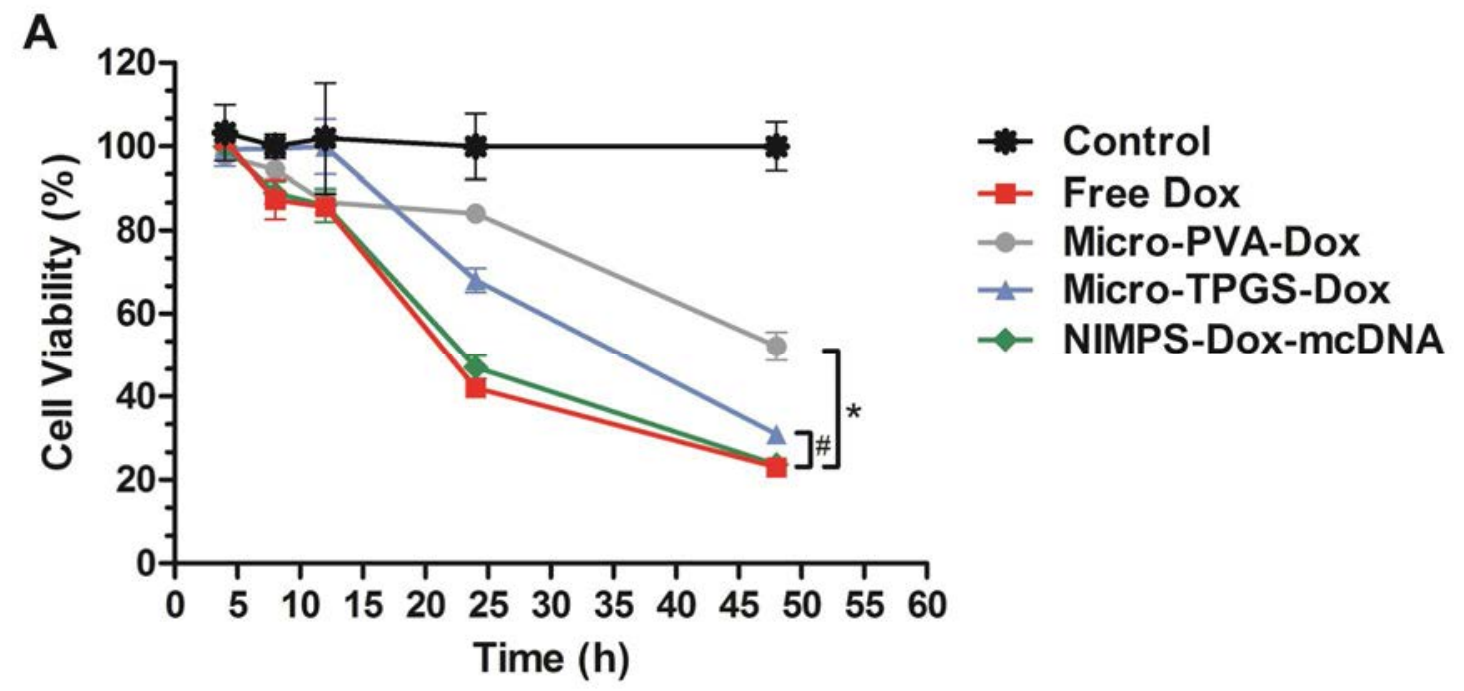

Figure 5. Cytotoxic activity of single-loadedmicrocarriers and dual-loaded delivery systems (micro-TPGS-Dox/CH-HR-mcDNA) in HeLa cancer cells. Control: non-treatedHeLa cells. Particles were administered at a dose of $200 \mu \mathrm{g} \cdot \mathrm{mL}^{-1}$. Data is represented as mean \pm s.d., $n=$ $5,{ }^{*} \mathrm{p}<0.05,{ }^{*} \mathrm{p}<0.01$ 
As the depicted in Figure 5micro-TPGS-Dox carriers display 1.7-fold higher cytotoxicity in comparison to their PLGA-PVA equivalents. Similarly, NIMPS exhibit a significantly higher cytotoxic activity when compared with single loaded micro-TPGS-Doxparticles (Figure 5; ${ }^{\#} \mathrm{p}<0.01$ ). Taken together, the results indicate thatthe inclusion of TPGS enhances the overall biological performance and cytotoxic activity of gas-generating PLGA microcarriers. Also, the encapsulation of mcDNA in the form of $\mathrm{CH}-\mathrm{HR}$ polyplexes originated transgene expression of mcDNA non-viralvectors, which are reported to be more effective than standard DNA both in vitro and in vivo[9].From a therapeutic point of view this is a crucial factor since cancer treatment may be improved by using mcDNA vectors that encode anti-tumoral genes (e.g. P53 or TRAIL $[34,35])$, and combining their action with the cytotoxic activity of chemotherapeutics, to achieve a synergistic anti-tumoral effect.

PLGA-NaHCO 3 -PVAbased microspheres for Doxdelivery to cancer cells have been previously described in the literature[25]. Herein, we have taken advantage of this simple and cost-effective technology to formulate $\mathrm{pH}$-responsive microspheres with further improved cellular uptake, and also demonstrated the possibility to efficiently co-delivery nanoparticlemcDNA in these systems. Due to its stability NIMPS provide the possibility to be locally delivered to cervix cancer location and respond to the acidic $\mathrm{pH}$ of this tumor[36, 37]. This is a major advantage when compared as example to other nanoparticulated PLGA-based systems (PLGA-PEI) for co-delivery and which require chemical modifications for being able toencapsulate drugs and genes and are unable to respond to extracellular stimuli [38]. The micron-size of the NIMPS system may prove beneficial in the future for inclusion in tandem or ovoid devices that are generally used in the clinics to locallyapplytreatment for this particular type of cancer [39]. In addition, the recently described potential of using gas generation fortheranostic applications based in ultrasound imaging further expands the applicability of these carriers [40].

\section{Conclusions}


In summary, in this study we have developed a pH-responsive nanoparticle-in-microsphere hybrid delivery system comprised by gas-generating PLGA-TPGS-Dox microcarriers and byamino acid chitosan nanoparticles loaded with mcDNA vectors. This multipart delivery platform has proven to respond to acidic $\mathrm{pH}$ and release its cargo in a spatiotemporally controlled mode. micro-TPGS-Dox microspheres have also displayed an improved cellular uptake in comparison to standard PLGA-PVA microcarriers. Adding to this, the concept of using nanoparticles inside microspheres for combinatorial therapy was supported by the evidence that the resulting NIMPS formulations have the highest cellular uptake, accomplish transgene expression, and above all exhibit an improved cytotoxic activity. It is also important to emphasize that to the best of our knowledge this was the first time that mcDNA-loaded nanoparticles were included in PLGA microspheres as a strategy to co-deliver anti-tumoral drugs and genetic material. NIMPS are in fact a valuable addition to the relatively few number of delivery systems that are currently capable of delivering DNA minicircles and chemotherapeutics simultaneously. In fact, considering the drawbacks associated with standalone cancer treatments such as those based on single drug administration these findings encourage the applicability of this hybrid system for exploring new drug-gene combinations and to bridge the gap between pre-clinical research and realistic clinical application.

In the future the versatility of PLGA microspheres to encapsulate both hydrophilic/hydrophobic drugs and the possibility of including cancer cell targeting biomolecules in TPGS structure will surely prove to be beneficial for the development of a delivery platform that is based on FDA approved materials. Additionally, 3D tumor spheroids can be used in the future for testing the therapeutic efficiency of NIMPS in high-throughput models that more closely mimic in vivo tumors.

\section{Acknowledgments}

The authors would like to acknowledgeSónia Miguel for the help in the acquisition of SEM images. This work was supported by the Portuguese Foundation for Science and Technology (FCT), (PEst-C/SAU/UI0709/2011and PEst-OE/SAU/UI0709/2014)).Vítor M. Gaspar 
acknowledges an individual PhD fellowship from FCT (SFRH/BD/80402/2011). The third author acknowledges a fellowship (CENTRO-07-ST24-FEDER-002014) from “ProgramaOperacional Regional do Centro 2007-2013 QREN" ("Mais Centro” program). 


\section{References}

[1] S. Mitragotri, P.A. Burke, R. Langer, Overcoming the challenges in administering biopharmaceuticals: formulation and delivery strategies, Nature Reviews Drug Discovery, 13 (2014) 655-672.

[2] A. Jhaveri, P. Deshpande, V. Torchilin, Stimuli-sensitive nanopreparations for combination cancer therapy, Journal of Controlled Release, 190 (2014) 352-370.

[3] J. Li, Y. Guo, Y. Kuang, S. An, H. M a, C. Jiang, Choline transporter-targeting and co-delivery system for glioma therapy, Biomaterials, 34 (2013) 9142-9148.

[4] Y. Wang, S. Gao, W.-H. Ye, H.S. Yoon, Y.-Y. Yang, Co-delivery of drugs and DNA from cationic coreshell nanoparticles self-assembled from a biodegradable copolymer, Nature materials, 5 (2006) 791796.

[5] S.W. Morton, M.J. Lee, Z.J. Deng, E.C. Dreaden, E. Siouve, K.E. Shopsowitz, N.J. Shah, M.B. Yaffe, P.T. Hammond, A Nanoparticle-Based Combination Chemotherapy Delivery System for Enhanced Tumor Killing by Dynamic Rewiring of Signaling Pathways, 2014.

[6] B. Yu, C. Tang, C. Yin, Enhanced antitumor efficacy of folate modified amphiphilic nanoparticles through co-delivery of chemotherapeutic drugs and genes, Biomaterials, 35 (2014) 6369-6378.

[7] D. de Melo-Diogo, V.M. Gaspar, E.C. Costa, A.F. Moreira, D. Markl, E. Gallardo, I.J. Correia, Combinatorial delivery of Crizotinib-Palbociclib-Sildenafil using TPGS-PLA micelles for improved cancer treatment, European Journal of Pharmaceutics and Biopharmaceutics, (2014).

[8] S.B. Uppada, T. Erickson, L. Wojdyla, D.N. Moravec, Z. Song, J. Cheng, N. Puri, Novel delivery system for T-oligo using a nanocomplex formed with an alpha helical peptide for melanoma therapy, International journal of nanomedicine, 9 (2014) 43.

[9] W.M. Dietz, N.E. Skinner, S.E. Hamilton, M.D. Jund, S.M. Heitfeld, A.J. Litterman, P. Hwu, Z-Y. Chen, A.M. Salazar, J.R. Ohlfest, M inicircle DNA is Superior to Plasmid DNA in Eliciting Antigenspecific CD8+T-cell Responses, M olecular Therapy, 21 (2013) 1526-1535.

[10] V.M. Gaspar, C. Gonçalves, D. de Melo-Diogo, E.C. Costa, J.A. Queiroz, C. Pichon, F. Sousa, I.J. Correia, Poly (2-ethyl-2-oxazoline)-PLA-g-PEI amphiphilic triblock micelles for co-delivery of minicircle DNA and chemotherapeutics, Journal of Controlled Release, 189 (2014) 90-104.

[11] Q. Zhang, R. Ran, L. Zhang, Y. Liu, L. Mei, Z. Zhang, H. Gao, Q. He, Simultaneous delivery of therapeutic antagomirs with paclitaxel for the management of metastatic tumors by a $\mathrm{pH}$-responsive anti-microbial peptide-mediated liposomal delivery system, Journal of Controlled Release, (2014).

[12] V. Gaspar, D.d. Melo-Diogo, E. Costa, A. Moreira, J. Queiroz, C. Pichon, I. Correia, F. Sousa, Minicircle DNA vectors for gene therapy: advances and applications, Expert Opinion on Biological Therapy, 0 1-27.

[13] D. Kobelt, M. Schleef, M. Schmeer, J. Aumann, P.M. Schlag, W. Walther, Performance of High Quality M inicircle DNA for In Vitro and In Vivo Gene Transfer, M olecular Biotechnology, (2012) 1-10.

[14] J.V. Natarajan, C. Nugraha, X.W. Ng, S. Venkatraman, Sustained-release from nanocarriers: a review, Journal of Controlled Release, 193 (2014) 122-138.

[15] Y. Wang, M.S. Shim, N.S. Levinson, H.W. Sung, Y. Xia, Stimuli-Responsive Materials for Controlled Release of Theranostic Agents, Advanced Functional M aterials, (2014).

[16] N.R. Ko, J. Cheong, A. Noronha, C.J. Wilds, J.K. Oh, Reductively-sheddable cationic nanocarriers for dual chemotherapy and gene therapy with enhanced release, Colloids and Surfaces B: Biointerfaces, (2014).

[17] G. Saravanakumar, W.J. Kim, Stimuli-Responsive Polymeric Nanocarriers as Promising Drug and Gene Delivery Systems, Intracellular Delivery II, Springer2014, pp. 55-91.

[18] M. Cai, M. Leng, A. Lu, L. He, X. Xie, L. Huang, Y. Ma, J. Cao, Y. Chen, X. Luo, Synthesis of amphiphilic copolymers containing zwitterionic sulfobetaine as $\mathrm{pH}$ and redox responsive drug carriers, Colloids and Surfaces B: Biointerfaces, (2014).

[19] J. Liu, Y. Huang, A. Kumar, A. Tan, S. Jin, A. M ozhi, X.-J. Liang, Ph-sensitive nano-systems for drug delivery in cancer therapy, Biotechnology advances, (2013). 
[20] J. Liu, H. Ma, T. Wei, X.-J. Liang, CO 2 gas induced drug release from pH-sensitive liposome to circumvent doxorubicin resistant cells, Chemical Communications, 48 (2012) 4869-4871.

[21] V. Gaspar, J. Marques, F. Sousa, R. Louro, J. Queiroz, I. Correia, Biofunctionalized nanoparticles with pH-responsive and cell penetrating blocks for gene delivery, Nanotechnology, 24 (2013) 275101.

[22] Z. Zhang, S.-S. Feng, The drug encapsulation efficiency, in vitro drug release, cellular uptake and cytotoxicity of paclitaxel-loaded poly(lactide)-tocopheryl polyethylene glycol succinate nanoparticles, Biomaterials, 27 (2006) 4025-4033.

[23] V.M. Gaspar, E.C. Costa, J.A. Queiroz, C. Pichon, F. Sousa, I.J. Correia, Folate-Targeted Multifunctional Amino Acid-Chitosan Nanoparticles for Improved Cancer Therapy, Pharm Res, (2014) 1-16.

[24] J.G. Marques, V.M. Gaspar, D. M arkl, E.C. Costa, E. Gallardo, I.J. Correia, Co-delivery of Sildenafil (Viagra ${ }^{\circledR}$ ) and Crizotinib for Synergistic and Improved Anti-tumoral Therapy, Pharm Res, (2014) 1-13.

[25] C.-J. Ke, W.-L. Chiang, Z.-X. Liao, H.-L. Chen, P.-S. Lai, J.-S. Sun, H.-W. Sung, Real-time visualization of pH-responsive PLGA hollow particles containing a gas-generating agent targeted for acidic organelles for overcoming multi-drug resistance, Biomaterials, 34 (2013) 1-10.

[26] L. Mu, P.-H. Seow, S.-N. Ang, S.-S. Feng, Study on surfactant coating of polymeric nanoparticles for controlled delivery of anticancer drug, Colloid and Polymer Science, 283 (2004) 58-65.

[27] M.J. Ernsting, M. Murakami, A. Roy, S.-D. Li, Factors controlling the pharmacokinetics, biodistribution and intratumoral penetration of nanoparticles, Journal of Controlled Release, 172 (2013) 782-794.

[28] S.A. Kulkarni, S.-S. Feng, Effects of particle size and surface modification on cellular uptake and biodistribution of polymeric nanoparticles for drug delivery, Pharm Res, 30 (2013) 2512-2522.

[29] A.F. M oreira, V.M. Gaspar, E.C. Costa, D. de M elo-Diogo, P. M achado, C.M. Paquete, I.J. Correia, Preparation of end-capped $\mathrm{pH}$-sensitive mesoporous silica nanocarriers for on-demand drug delivery, European Journal of Pharmaceutics and Biopharmaceutics, (2014).

[30] J.S. Fordtran, S.G. Morawski, C.A. Santa Ana, F. Rector Jr, Gas production after reaction of sodium bicarbonate and hydrochloric acid, Gastroenterology, 87 (1984) 1014-1021.

[31] M. Keeney, S.-G. Ong, A. Padilla, Z. Yao, S. Goodman, J.C. Wu, F. Yang, Development of Poly ( $\beta$ amino ester)-Based Biodegradable Nanoparticles for Nonviral Delivery of M inicircle DNA, ACS nano, 7 (2013) 7241-7250.

[32] C. Zhang, S. Gao, W. Jiang, S. Lin, F. Du, Z. Li, W. Huang, Targeted minicircle DNA delivery using folate-poly (ethylene glycol)-polyethylenimine as non-viral carrier, Biomaterials, 31 (2010) 60756086.

[33] S. Jain, M. Amiji, Nanoparticles-in-Microsphere Oral Delivery Systems (NiM OS) for Nucleic Acid Therapy in the Gastrointestinal Tract, Mucosal Delivery of Biopharmaceuticals, Springer2014, pp. 283-312.

[34] E. Pazarentzos, N.D. Mazarakis, Anticancer Gene Transfer for Cancer Gene Therapy, Anticancer Genes, Springer2014, pp. 255-280.

[35] T.H. Kim, H.H. Jiang, C.W. Park, Y.S. Youn, S. Lee, X. Chen, K.C. Lee, PEGylated TNF-related apoptosis-inducing ligand (TRAIL)-loaded sustained release PLGA microspheres for enhanced stability and antitumor activity, Journal of Controlled Release, 150 (2011) 63-69.

[36] B.A. Webb, M. Chimenti, M .P. Jacobson, D.L. Barber, Dysregulated pH: a perfect storm for cancer progression, Nature Reviews Cancer, 11 (2011) 671-677.

[37] G. Schwickert, S. Walenta, K. Sundfør, E.K. Rofstad, W. Mueller-Klieser, Correlation of high lactate levels in human cervical cancer with incidence of metastasis, Cancer research, 55 (1995) 4757-4759.

[38] W.-P. Su, F.-Y. Cheng, D.-B. Shieh, C.-S. Yeh, W.-C. Su, PLGA nanoparticles codeliver paclitaxel and Stat3 siRNA to overcome cellular resistance in lung cancer cells, International journal of nanomedicine, 7 (2012) 4269.

[39] L. Fokdal, K. Tanderup, S.B. Hokland, L. Røhl, E.M. Pedersen, S.K. Nielsen, M. Paludan, J.C. Lindegaard, Clinical feasibility of combined intracavitary/interstitial brachytherapy in locally 
advanced cervical cancer employing MRI with a tandem/ring applicator in situ and virtual preplanning of the interstitial component, Radiotherapy and Oncology, 107 (2013) 63-68.

[40] K.H. M in, H.S. M in, H.J. Lee, D.J. Park, J.Y. Yhee, K. Kim, I.C. Kwon, S.Y. Jeong, O.F. Silvestre, X. Chen, Y.-S. Hwang, E.-C. Kim, S.C. Lee, pH-Controlled Gas-Generating M ineralized Nanoparticles: A Theranostic Agent for Ultrasound Imaging and Therapy of Cancers, ACS Nano, (2015).

Graphical abstract

\section{Nanoparticle-in-Microspheres (NIMPS)}

Chitosan-mcDNA

Nanoparticles

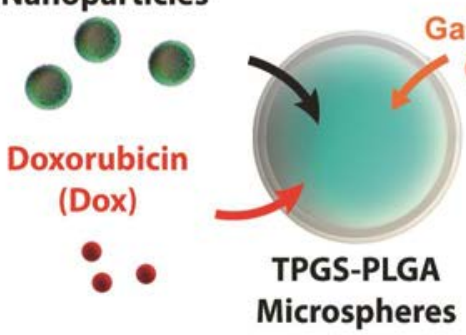

Gas-generating

$\left(\mathrm{NaHCO}_{3}\right)$

Acidic $\mathrm{pH}$

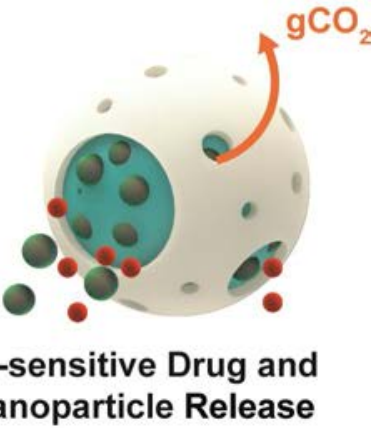

